\title{
Application of Electrodynamic Catalytic Reactors for Intensification of Heat and Mass Exchange Processes of Heterophase Catalysis
}

\author{
EKATERINA A. SHULAEVA \\ Department "Automated Technology and Information Systems" \\ Branch of the Ufa State Petroleum Technological University in Sterlitamak \\ Oktyabrya Ave., 2.Sterlitamak, 453118 Bashkortostan Republic \\ RUSSIA
}

\begin{abstract}
This article discusses a model of an electrodynamic reactor. It is fundamentally different in the way of supplying energy to the reaction zone from the reactors currently in operation in industry. This significantly reduces energy consumption, simplifies process control and increases the efficiency of the reactor. The environmental advantage of the developed reactor is also of great importance. There are no emissions of gases into the atmosphere, which are formed in large quantities during fuel combustion in superheating furnaces, the consumption of water is reduced, which is used only in a closed cycle to cool the microwave generator and circulator as a matching load during its operation. The overall efficiency of the electrodynamic reactor is 1.2 times higher than that of existing industrial ones. A method for calculating thermodynamic processes in electrodynamic reactors is proposed. It allows you to determine the technological parameters of the process to ensure a given temperature distribution and provides the maximum yield of the target reaction products with the minimum possible energy consumption of electromagnetic radiation.
\end{abstract}

Key-Words: - microwave, electrodynamic reactor, catalyst, temperature distribution, heterophase catalysis, heat and mass transfer

Received: March 19, 2021. Revised: January 3, 2022. Accepted: February 8, 2022. Published: March 2, 2022.

\section{Introduction}

The creation of energy-saving and resource-saving technologies for the rational use of natural resources in the petrochemical industry is a very urgent problem. One of the ways to solve which is the use of various physical methods of influencing technological environments, in particular, electromagnetic radiation of the microwave range, as one of the effective methods of energy transfer.

The use of microwave as a heat carrier for heating media of various natures is one of the ways to increase the efficiency of modern chemical production. It is stimulating research on the use of microwave in chemical technology. Currently, in Russia, the USA, UK, Canada, Japan and other countries of the world, there are chemical laboratories focused on research in this field of chemistry and on the creation of special microwave units for carrying out specific chemical processes.

The use of microwave in the chemical and petrochemical industry is constrained by the lack of reliable data on the specifics of chemical transformations occurring under the action of microwave taking into account the specific nature of energy transfer in the reaction zone and due to the lack of methods for calculating technological units and reaction devices that use this type of energy transfer. It is also necessary to take into account that the efficiency of transformation of the energy of the electromagnetic field into heat, which is necessary for carrying out chemical transformations. First of all, this is determined by the properties of the technological environment, which is a heat converter [1]. Therefore, the study of the effect of electromagnetic radiation in the microwave range on various technological environments requires the introduction of additional parameters that determine the efficiency of conversion of electromagnetic energy into thermal energy. This is an important both theoretical and practical task aimed at optimal design of technological cycles of oil refining and operation of reaction devices and technological units.

\section{Problem Formulation}

The main feature when calculating an electrodynamic reactor, in comparison with a traditional catalytic reactor, is the need to take into account the properties of the electromagnetic field and the electrophysical properties of the catalyst substance. These parameters are directly related to 
the determination of the characteristic dimensions of the reaction unit, i.e. its diameter and height [2].

Another important characteristic determined by the parameters of the electromagnetic field and affecting the design parameters of the reactor is the minimum diameter of the reaction device, it is determined from the wavelength of electromagnetic radiation. When using microwave the body of the reaction device is a resonator. The main condition for the propagation of electromagnetic waves in the resonator is the ratio [3]:

$$
\lambda<\lambda_{c r} \text {, }
$$

where $\lambda$ - wavelength of electromagnetic radiation, $\mathrm{m} ; \lambda_{c r}-$ critical wavelength, $\mathrm{m}$.

The critical wavelength for waves of the $E_{m n}$ type is determined by the following relation:

$$
\lambda_{\mathrm{crE}}=\frac{2 \pi a}{P_{m n}},
$$

where $a$ is the radius of the waveguide, $\mathrm{m} ; P_{m n}$ are the roots of the Bessel function $J_{m}$ of order $m$, the values of which are given in Table 1 [4].

Table 1. Roots of the Bessel function $J_{m}$ of order $m$

\begin{tabular}{|c|c|c|c|}
\hline \multirow{2}{*}{$m$} & \multicolumn{3}{|c|}{$n$} \\
\cline { 2 - 4 } & 1 & 2 & 3 \\
\cline { 2 - 4 } & \multicolumn{3}{|c|}{$P_{m n}$} \\
\hline 0 & 2,405 & 5,52 & 8,654 \\
\hline 1 & 3,832 & 7,016 & 10,173 \\
\hline 2 & 5,136 & 8,417 & 11,620 \\
\hline
\end{tabular}

Thus, the radius of the reaction units can be determined:

$$
a>\frac{\lambda P_{m n}}{2 \pi} \text {. }
$$

Similarly, you can determine the critical wavelength for waves of the $H_{m n}$ type:

$$
\lambda_{\mathrm{cr} \mathrm{H}}=\frac{2 \pi a}{P_{m n}{ }^{\prime}},
$$

where $P_{m n}^{\prime}$ are the roots of the Bessel function $J_{m}{ }^{\prime}$, the values of which are given in Table 2 [4].

Table 2. Roots of the Bessel function $J_{m}$ 'of order

\begin{tabular}{|c|c|c|c|}
\hline \multirow{2}{*}{$m$} & \multicolumn{3}{|c|}{$m$} \\
\cline { 2 - 4 } & 1 & 2 & 3 \\
\cline { 2 - 4 } & \multicolumn{3}{|c|}{$P_{m n}{ }^{\prime}$} \\
\hline 0 & 3,832 & 7,016 & 10,174 \\
\hline 1 & 1,841 & 5,332 & 8,536 \\
\hline 2 & 3,054 & 6,705 & 9,965 \\
\hline
\end{tabular}

Thus, the minimum diameter of the reaction device, in which electromagnetic waves of the $E_{01}$ and $H_{11}$ types can propagate without loss at a wavelength of $0.12 \mathrm{~m}$, corresponding to a frequency of $2450 \mathrm{~Hz}$, is $0.092 \mathrm{~m}$.

Based on the required capacity of the reactor, the volume of the catalyst is determined, taking into account the limitations stated above. Thus:

$$
V_{K}=\frac{V_{G}}{W},
$$

where $V_{K}$ is the catalyst volume, $\mathrm{m}^{3} ; V_{G}-$ gas volumetric velocity, $\mathrm{m}^{3} / \mathrm{h} ; W$ - volumetric feed rate, $\mathrm{h}^{-1}$.

To assess the energy efficiency of the catalytic process under the action of microwave, the thermal balance of a microwave unit is calculated by the formula:

$$
Q_{m}+Q_{\text {in }}=Q_{p}+Q_{o u t}+Q_{l o s},
$$

where $Q_{m}$ is the heat released during the absorption of electromagnetic radiation, $\mathrm{J} / \mathrm{h} ; Q_{i n}$ is the heat introduced into the reaction plant by the flow of the gas mixture, $\mathrm{J} / \mathrm{h}$, defined as $Q_{i n}=G \cdot c \cdot T_{i n}$, where $G$ is the amount of substance entering the reactor, $\mathrm{mol} / \mathrm{h}, c$ is the molar heat capacity, $\mathrm{J} /(\mathrm{mol} \bullet \mathrm{K}), T_{\text {in }}$ is the temperature of the gas mixture at the entrance to the reactor, $\mathrm{K} ; Q_{p}$ - heat absorbed / released during chemical reactions, $\mathrm{J} / \mathrm{h} ; Q_{o u t}$ is the heat carried away from the reaction plant, $\mathrm{J} / \mathrm{h} ; Q_{l o s}$ - heat losses in the reaction plant, $\mathrm{J} / \mathrm{h}$, as a rule, for the purposes of technological calculation is taken at 5\%, of the heat entering the reaction plant.

Thus, the thermal efficiency of the reaction plant:

$$
\eta=\frac{Q_{p}}{Q_{m}} .
$$

The power of the magnetron required for carrying out chemical transformations can be calculated based on the heat balance equation of the reaction plant (6):

$$
Q_{m}=Q_{p}+Q_{\text {out }}-Q_{\text {in }}+Q_{\text {los }} \text {. }
$$

Thus, expressions (1-7) allow for the technological calculation of the reaction device. The peculiarities of this calculation include the need to determine the "total" depths of microwave absorption for the catalysts used in each specific technological process on the basis of experimental data. Another feature is the need to select the diameter of the reaction device, based on the wavelength of the microwave generator.

Reactor design for carrying out processes under microwave. On the basis of laboratory research, a special reaction device was developed for the implementation of endothermic processes using microwave heating [5]. The schematic diagram of the reaction device (electrodynamic reactor) is 
shown in Fig. 1. The reactor has an inner diameter of $100 \mathrm{~mm}$, a maximum height of $1340 \mathrm{~mm}$, and a wall thickness of $5 \mathrm{~mm}$. The installation includes a continuous microwave source with a fixed oscillation frequency of $2450 \mathrm{MHz}$ and an adjustable output power of $0-5 \mathrm{~kW}$.

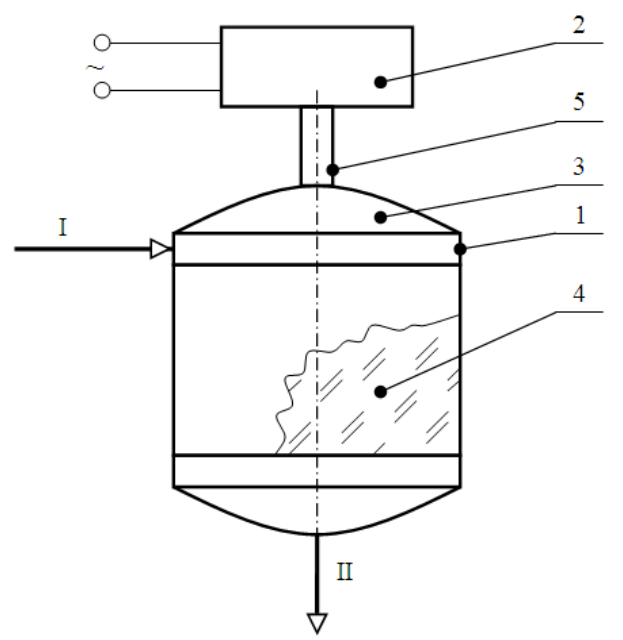

Fig. 1: Schematic diagram of a microwave catalytic reactor for endothermic heterophase reactions: 1 reactor vessel; 2 - microwave generator; 3 - top cover of the reactor; 4 - catalyst; 5 - waveguide; I raw material; II - contact gas.

The reactor (resonator for a microwave generator) is a vertical cylindrical thermally insulated vessel and consists of three main units (Figure 2): input of raw materials and microwave radiation, a reaction zone and a unit for output of reaction products.

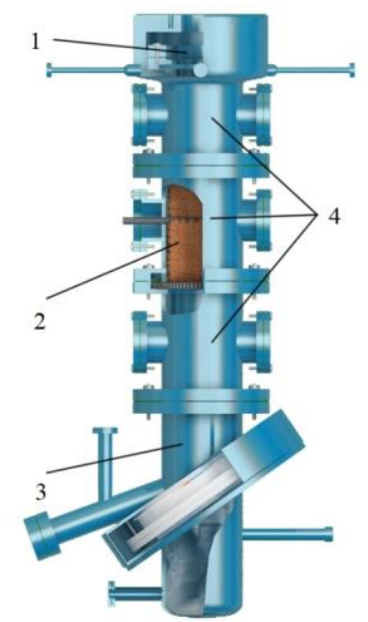

Fig. 2: Model of an electrodynamic reactor in section: 1 - input unit; 2 - catalyst; 3 - output node; 4 - reaction units.

The upper cover of the reactor plays the role of a radiating antenna that ensures a uniform distribution of energy over the cross section of the reactor. Alloy steel resistant to corrosive media in a wide temperature range and having sufficient electrical conductivity, is proposed as a material for the manufacture of the reactor. The last condition is necessary to prevent losses of electromagnetic energy.

The fluoroplastic membrane is installed in the injection unit, which ensures the tightness of the reaction zone. The raw material is introduced into the reaction chamber with tangential input. The outgoing gas is discharged from the bottom. Distribution grids for the catalyst are installed in the reaction chamber they are made of heat-resistant ceramics permeable to microwaves.

A matching chamber for absorbing residual radiation is located in the reaction product outlet. Residual radiation due to its incomplete absorption by the catalyst substance penetrates through the membrane of the matching chamber and is absorbed by water. The inclined surface of the matching chamber attenuates the direct reflection of microwave radiation into the magnetron. In the matching chamber, crushed catalyst particles are collected.

The matching chamber is equipped with a hatch by means of the fittings it is possible to regulate the level of filling the chamber with liquid. Control and measurement devices are mounted on the hatch cover [6].

The advantages of the reactor include the ability to operate in a wide temperature range and resistance to aggressive media. Alloy steel was chosen as the structural material of the reactor, which has proven itself in the petrochemical industry for the manufacture of various apparatus and equipment.

Through modular construction of the reaction zone may change the size of the reactor, while maintaining the desired ratio of height to diameter. This ratio can vary from minimum values to 9.75 .

At present, the use of electromagnetic radiation of the microwave range is becoming more and more widespread for carrying out various physical transformations as a way of more efficient, from the standpoint of energy consumption per unit of the product obtained, and faster heating of substances of various nature, which is a new direction in the creation of energy and resource-saving technologies.

In contrast to the traditional convective mechanism of heat transfer, when using microwave heating, electromagnetic radiation, being absorbed by the substance, leads to the volumetric dissipation of the energy of the electromagnetic field in the substance and, as a consequence, to the volumetric 
heating of the latter, which significantly reduces the heating time and gives a greater uniformity of heating.

The dissipated energy depends both on the parameters of the electromagnetic field (frequency, amplitude of the electric and magnetic field strength vectors) and on the electrophysical properties of the substance (real and imaginary parts of the dielectric and magnetic permeability, conductivity of the medium).

The use of this heating method has a number of significant advantages, in particular, the use of heat carriers is excluded, which ensures cleanliness, there is no need for separation, and the mass of the process stream is reduced. Also, this type of heating has low inertia, which facilitates process control and increases safety. Therefore, the creation of reaction devices, in which the role of the coolant is played by electromagnetic radiation of the microwave range, is a very promising task.

\section{Problem Solution}

The model of functioning of an electrodynamic reactor (Fig. 3) is as follows [2]: when microwave is absorbed by a catalyst substance, volumetric heat sources appear in it, the power density of which $\left(\mathrm{W} / \mathrm{m}^{3}\right)$ is determined by the expression:

$$
q_{v}(x)=\frac{P}{F \delta_{E}(1-\varepsilon)} \exp \left\{-K \frac{x}{\delta_{E}}\right\},
$$

where $P$ is the power of electromagnetic radiation absorbed by the catalyst substance, W; $F$ is the cross-sectional area of the reactor, $\mathrm{m}^{2} ; \delta_{E}-$ "total" depth of absorption of electromagnetic radiation, $\mathrm{m}$; $\varepsilon$ is the porosity of the catalyst layer; $K$ is a dimensionless coefficient that depends on the physical properties of the material and takes into account the ability of the substance to absorb electromagnetic energy; $x$ - coordinate directed along the reactor axis.



Fig. 3: Diagram of an electrodynamic reactor: $1-$ gas phase; 2 - solid phase; 3 - contact gas.
One of the important tasks in the design of a reactor plant of this class is to find the temperature distribution in the solid and gas phase when the gaseous medium is blown through the catalyst bed with a mass flow rate $Q$ under microwave heating conditions in a one-dimensional approximation, we investigate two heat balance equations: the first equation is the heat balance gas and solid phase, the second is the heat balance of the solid phase for the elementary volume of the reactor.

For gas and solid:

$$
\begin{aligned}
& -\frac{\partial}{\partial t}\left(c_{p g} \rho_{g} \varepsilon T_{g}+c_{s} \rho_{s}(1-\varepsilon) T_{s}\right)= \\
& =-\frac{\partial}{\partial x}\left(\lambda_{g} \frac{\partial T_{g}}{\partial x}+\lambda_{s} \frac{\partial T_{s}}{\partial x}\right)+c_{p g} Q \frac{\partial T_{g}}{\partial x}-q_{v},
\end{aligned}
$$

where $c_{p g}$ is the averaged isobaric heat capacity of the gas phase, $\mathrm{J} /(\mathrm{kg} \bullet \mathrm{K}) ; c_{s}$ is the averaged heat capacity of the solid phase, $\mathrm{J} /(\mathrm{kg} \cdot \mathrm{K}) ; T_{g}-$ gas temperature, $T_{s}$ - solid phase temperature, $\varepsilon-$ porosity, $\lambda_{\mathrm{g}}$ - effective thermal conductivity of gas, $\mathrm{W} /(\mathrm{m} \cdot \mathrm{K}) ; \lambda_{\mathrm{s}}-$ effective thermal conductivity of a solid, $\mathrm{W} /(\mathrm{m} \cdot \mathrm{K}) ; Q$ is the mass velocity of the gas phase, $\mathrm{kg} /(\mathrm{m} 2 \bullet \mathrm{s}) ; q_{v}-$ volumetric power of heat sources, $\mathrm{W} / \mathrm{m}^{3}$.

$$
\begin{aligned}
& -\frac{\partial}{\partial t}\left(c_{s}(1-\varepsilon) \rho_{s} T_{s}\right)= \\
& =-\frac{\partial}{\partial x}\left(\lambda_{s} \frac{\partial T_{s}}{\partial x}\right)+\alpha\left(T_{s}-T_{g}\right)-q_{v},
\end{aligned}
$$

where $\alpha$ is the effective volumetric heat transfer coefficient, $\mathrm{W} /\left(\mathrm{m}^{3} \cdot \mathrm{K}\right)$.

Simplifying the last equations, we get:

$$
\begin{aligned}
& -c_{p g} \rho_{g} \varepsilon \frac{\partial T_{g}}{\partial t}-c_{s} \rho_{s}(1-\varepsilon) \frac{\partial T_{s}}{\partial t}= \\
& =-\lambda_{g} \frac{\partial^{2} T_{g}}{\partial x^{2}}-\lambda_{s} \frac{\partial^{2} T_{s}}{\partial x^{2}}+c_{p g} Q \frac{\partial T_{g}}{\partial x}-q_{v} \\
& -c_{s} \rho_{s}(1-\varepsilon) \frac{\partial T_{s}}{\partial t}= \\
& =-\lambda_{s} \frac{\partial^{2} T_{s}}{\partial x^{2}}+\alpha\left(T_{s}-T_{g}\right)-q_{v}
\end{aligned}
$$

Subtracting (12) from (13), we obtain the following relations connecting the temperatures of the solid $T_{s}$ and gas $T_{g}$ phases:

$$
\begin{aligned}
& -c_{p g} \rho_{g} \varepsilon \frac{\partial T_{g}}{\partial t}=-\lambda_{g} \frac{\partial^{2} T_{g}}{\partial x^{2}}+c_{p g} Q \frac{\partial T_{g}}{\partial x}- \\
& -\alpha\left(T_{s}-T_{g}\right)
\end{aligned}
$$

Thus, to find the temperature distribution of the gas and the solid phase over the height of the 
electrodynamic reactor, it is necessary to solve the system of equations (12), (14).

Expressions (12) and (13) are the solution to the system of equations (12), (14).

The temperature of the gas phase in an electrodynamic reactor can be determined from the following expression:

$$
\begin{aligned}
& T_{g}=C_{1} \exp \left\{\frac{\frac{c_{p g} Q}{\lambda_{g}}+\sqrt{\left(\frac{c_{p g} Q}{\lambda_{g}}\right)^{2}+4 \frac{\alpha}{\lambda_{g}}}}{2} x\right\}+ \\
& +C_{2} \exp \left\{\frac{\frac{c_{p g} Q}{\lambda_{g}}-\sqrt{\left(\frac{c_{p g} Q}{\lambda_{g}}\right)^{2}+4 \frac{\alpha}{\lambda_{g}}}}{2} x\right\}-
\end{aligned}
$$

$$
-\frac{2}{\sqrt{\left(\frac{c_{p g} Q}{\lambda_{g}}\right)^{2}+4 \frac{\alpha}{\lambda_{g}}}} \int_{0}^{x} \frac{\alpha}{\lambda_{g}} T_{s}(\xi) \exp \left\{\frac{c_{p g} Q}{2 \lambda_{g}}(x-\xi)\right\} \times
$$

$$
\times \operatorname{sh}\left(\frac{\sqrt{\left(\frac{c_{p g} Q}{\lambda_{g}}\right)^{2}+4 \frac{\alpha}{\lambda_{g}}}}{2}(x-\xi)\right) d \xi,
$$

where $C_{1}$ and $C_{2}$ are the integration constants obtained from the boundary conditions of the problem.

The temperature of the solid phase in an electrodynamic reactor can be determined from the following expression:

$$
\begin{aligned}
& T_{s}=C_{1} \operatorname{ch}\left(\sqrt{\frac{\alpha}{\lambda_{s}}} x\right)+C_{2} \operatorname{sh}\left(\sqrt{\frac{\alpha}{\lambda_{s}}} x\right)- \\
& -\frac{1}{\sqrt{\frac{\alpha}{\lambda_{s}}}} \int_{0}^{x}\left(\frac{\alpha}{\lambda_{s}} T_{g}(\xi)+\frac{q_{v}(\xi)}{\lambda_{s}}\right) \times \\
& \times \operatorname{sh}\left(\sqrt{\frac{\alpha}{\lambda_{s}}}(x-\xi)\right) d \xi,
\end{aligned}
$$

where $C_{1}$ and $C_{2}$ are the integration constants obtained from the boundary conditions of the problem.

Thus, the solution of the system of equations (15) and (16) by the method of sequential approximation makes it possible to find the temperature distribution in the solid and gas phase along the height of the microwave reactor during purging of the chemically neutral gas phase.

Estimation of the coefficients of effective thermal conductivity and heat transfer. For the correct solution of the systems of equations described above, it is necessary to know the coefficients of effective thermal conductivity, effective heat transfer, as well as the coefficient of heat transfer to the side surface of the reactor.

To determine them, you can use the formulas that summarize the experimental studies that were carried out by a number of authors. These dependences have a satisfactory accuracy in finding the corresponding coefficients.

Effective thermal conductivity coefficient in a fixed catalyst bed. In [2], the following equations were experimentally established to find the effective thermal conductivity of a fixed catalyst bed:

for spherical granules:

$$
\frac{\lambda_{s}}{\bar{\lambda}_{g}}=\frac{1}{D_{p}}\left(\frac{\bar{\lambda}_{s}}{\bar{\lambda}_{g}}\right)^{0,12}\left(11,15+\frac{0,01915 d_{k} Q}{q \mu}\right),
$$

for cylindrical granules:

$$
\frac{\lambda_{s}}{\bar{\lambda}_{g}}=\frac{1}{D_{p}}\left(\frac{\bar{\lambda}_{s}}{\bar{\lambda}_{g}}\right)^{0,12}\left(11,97+\frac{0,0348 d_{k} Q}{\varepsilon \mu}\right),
$$

where $\bar{\lambda}_{g}$ is the coefficient of thermal conductivity of the gas, $\mathrm{W} /(\mathrm{m} \bullet \mathrm{K}) ; D_{\mathrm{p}}-$ reactor diameter, $\mathrm{m} ; \bar{\lambda}_{s}-$ thermal conductivity of the catalyst material, $\mathrm{W} /(\mathrm{m} \cdot \mathrm{K}) ; Q$ is the mass velocity of the gas phase, $\mathrm{kg} /(\mathrm{m} 2 \bullet \mathrm{s}) ; \varepsilon$ - layer porosity, $\mu$ - dynamic viscosity coefficient, Pa $\cdot \mathrm{s} ; d_{\mathrm{k}}$ is the diameter of the catalyst particles, $\mathrm{m}$ (in the case of cylindrical granules, $d_{k}=$ $\varphi d_{c}$, where $d_{c}$ is the cylinder diameter).

The sphericity coefficient $\varphi$ is the ratio of the surface of the equal-sized sphere $F_{s}$ to the surface of the catalyst granule $F_{g}$ :

$$
\varphi=\frac{F_{\mathrm{s}}}{\mathrm{F}_{\mathrm{g}}}=\left(\frac{d_{V}}{d_{F}}\right)^{2},
$$

where $d_{F}, d_{V}$ are the diameters of the balls, which are equivalent to the catalyst granule in terms of surface and volume.

For spherical granules $\varphi=1$, for cylindrical granules the values of $\varphi$ are presented in the table 3 .

Heat transfer coefficient from the particle surface. As a generalization of the experimental data in [2], an expression is given for determining the volumetric coefficient of effective heat transfer $\alpha$ in a fixed bed: 


$$
\frac{\alpha}{c_{p} Q}\left(\frac{c_{p} \mu}{\lambda_{g}}\right)^{\frac{2}{3}}=\frac{0,535}{(\varphi \operatorname{Re})^{0,3}-1,6}
$$

where $\operatorname{Re}=\frac{\sqrt{F_{k}} Q}{\mu(1-\varepsilon)}$ is the Reynolds criterion; $F_{k}$ is the surface area of the particle, $\varphi$ is the coefficient of sphericity of the particle.

Table 3. Sphericity coefficients for cylindrical catalyst granules

\begin{tabular}{|c|c|c|c|c|}
\hline \multirow{2}{*}{ Body } & \multicolumn{4}{|c|}{ Cylinder } \\
\cline { 2 - 5 } & $h=d / 2$ & $h=d / 6$ & $h=d / 20$ & $h=d / 30$ \\
\hline$\varphi$ & 0,827 & 0,594 & 0,323 & 0,220 \\
\hline \multirow{2}{*}{ Body } & \multicolumn{4}{|c|}{ Cylinder } \\
\cline { 2 - 5 } & $h=d$ & $h=1,5 d$ & $h=5 d$ & $h=10 d$ \\
\hline$\varphi$ & 0,438 & 0,860 & 0,691 & 0,580 \\
\hline
\end{tabular}

Heat transfer coefficient to the wall. To find the effective coefficient of heat transfer to the reactor wall, you can use the dependence proposed in [2], as follows:

$$
\alpha=3,6 \frac{\bar{\lambda}_{g}}{D_{p}}\left(\frac{d_{k} Q}{\mu \varepsilon}\right)^{0,365} .
$$

Thus, finding the effective heat transfer coefficients, the effective heat capacity in a fixed catalyst bed, the heat transfer coefficient from the side surface of the reactor complete the problem of finding the temperature distribution in the solid (catalyst) and gas (reaction mixture) phases over the height of the electrodynamic reactor.

Characteristic time of transition to stationary state. When simulating physicochemical processes in electrodynamic reactors according to the proposed mathematical model, which is described by the system of equations (12) - (15), only stationary modes of operation were considered, therefore, it becomes necessary to assess the time domain of application of these stationary solutions to non-stationary processes that can occur, according to for various reasons in these reaction devices.

Such an estimate can be made by a parameter called the characteristic time of transition to a stationary state, which allows, firstly, to qualitatively evaluate the influence of various parameters on the dynamic characteristics of such a system, and secondly, to determine the time domain of application of the stationary system of equations (12) - (15).

Let us estimate the characteristic time of establishment of a stationary state from the temperature distribution in the gas phase, which is decisive for finding the rate of a chemical reaction and, accordingly, the degree of conversion, for which we write the energy equation for the gas phase in the one-dimensional approximation for an elementary volume

$$
\begin{aligned}
& -c_{p g} \rho_{g} \varepsilon \frac{\partial T_{g}}{\partial t}=-\lambda_{e f, g} \frac{\partial^{2} T_{g}}{\partial x^{2}}+c_{p g} G \frac{\partial T_{g}}{\partial x}- \\
& -\alpha_{e f} a\left(T_{s}-T_{g}\right)
\end{aligned}
$$

Representing the last equation in a difference form, assuming that $\left(T_{s}-T_{g}\right) \sim \Delta T_{g}$. For the characteristic size, it is convenient to take the value $\delta_{E}$, which determines the depth of penetration of the electromagnetic wave into the substance, and, discarding the second-order quantities, we obtain

$$
c_{p g} \rho_{g} \varepsilon \frac{\Delta T_{g}}{\tau}=\alpha_{e f} a \Delta T_{g}-c_{p g} G \frac{\Delta T_{g}}{\delta_{E}},
$$

where $\tau-$ is the characteristic time of the onset of the stationary regime.

Thus, simplifying the last equation, we obtain

$$
\tau=\frac{c_{p g} \rho_{g} \varepsilon}{\alpha_{e f} a-\frac{c_{p g} G}{\delta_{E}}} \text {. }
$$

As can be seen from expression (24), an increase in the heat capacity of the gas leads to a significant increase in the time required to establish a stationary regime. An increase in the gas density also leads to an increase in the transition time to a stationary state, also to an increase in the transition time to a stationary state, and an increase in the porosity of the solid phase. The mass velocity of the gas phase also has a significant effect on the time of transition to the stationary regime. As can be seen from the obtained expression, with an effective heat transfer coefficient $\alpha_{e f} \sim G^{0,7}$, with an increase in the mass velocity, the denominator of the expression slightly decreases, which, in general, leads to an increase in the time of the onset of the stationary regime. An increase in the penetration depth also leads to an increase in the time of the onset of the stationary regime. Thus, we have proposed (24), which makes it possible to qualitatively assess the influence of various parameters on the characteristic time of the onset of a stationary state. For a more accurate assessment, it is necessary to solve non-stationary equations describing the physicochemical processes occurring in electrodynamic reactors, which may be a topic for further research in this area.

Numerical experiments were carried out according to the proposed model, the results of which are shown in the figures. Fig. 4 presents graphs showing the effect of the mass flow rate of 
the gas flow on the final temperature distribution in the solid and gas phases.

As can be seen from the graphs presented, at low values of the mass velocity of the gas phase, higher values of the steady-state temperature of the solid phase are observed, which is caused by a decrease in heat transfer from the heated catalyst to the blown gas phase. On the other hand, it becomes necessary to maintain optimal values of the mass rate to increase the yield of the target products during the course of the reaction. Thus, it is possible to determine the optimal values of the mass velocity when assessing the yield of the target product and the maximum value of the thermal efficiency of the electrodynamic reactor.

The influence of the porosity of the solid phase layer (Fig. 5) has a noticeable effect on the temperature distribution along the height of the reactor, primarily due to a decrease in the mass of the solid phase, while, with a constant penetration depth of electromagnetic radiation, a significant increase in the power of volumetric energy sources occurs, thereby a more intense heating the catalyst with greater porosity.

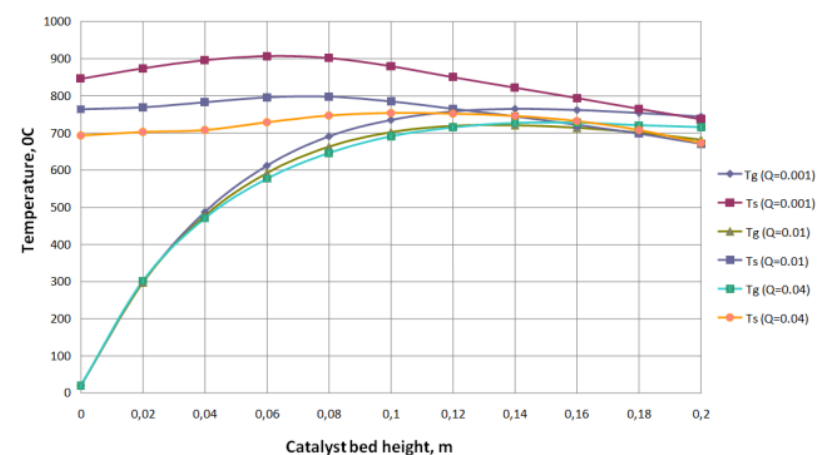

Fig. 4: Distribution of temperature in the solid and gas phase along the height of the electrodynamic reactor at different mass velocities of the gas flow.

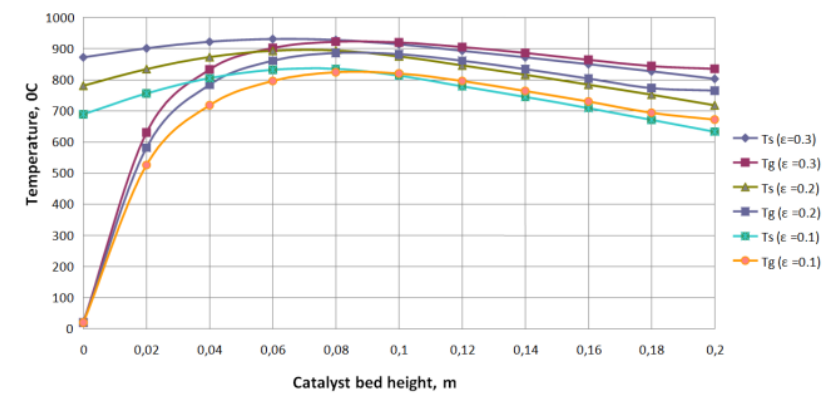

Fig. 5: Distribution of temperatures in the solid and gas phases along the height of the reactor at different porosities of the solid phase.

An assessment of the effect of the penetration depth of electromagnetic radiation into the catalyst substance on the final temperature distributions in the solid and gas phase is shown in Fig. 6. As can be seen from the graphs, an increase in the penetration depth $\delta$ leads to a decrease in the maximum temperatures in the solid phase and a decrease in the temperature gradient in the gas phase by at the initial stage with a shift of the maximum of the curves of the temperature profile of the solid and gas phases to the lower part of the reactor and, accordingly, a higher level of temperatures at the outlet of the reactor.

Based on these data, it can be concluded that the more efficient, from the standpoint of thermal efficiency, is the catalyst, which, other things being equal, has a smaller penetration depth of electromagnetic radiation at the same level of supplied energy, but it is necessary to take into account the fact that with a decrease in depth penetration, the temperature at the inlet to the reactor rises, which can lead to overheating of the upper layers of the catalyst, and, as a consequence, to its destruction.



Fig. 6: Temperature distribution in the solid and gas phases along the height of the reactor depending on the depth of penetration of electromagnetic radiation into the catalyst substance.

The influence of the gas thermal conductivity coefficient on the temperature distribution is illustrated by the graph shown in Fig. 7.

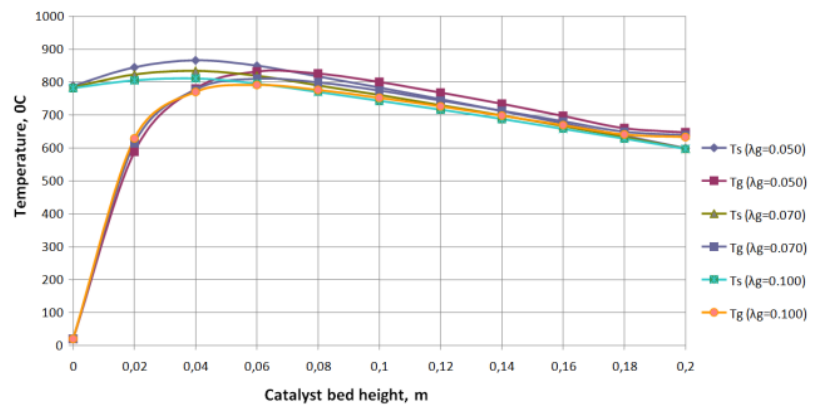

Fig. 7: Distribution of temperature in the solid and gas phase along the height of the reactor depending on the coefficient of thermal conductivity of the gas phase. 
At lower values of the gas phase thermal conductivity coefficient, with other parameters unchanged, a slightly higher temperature level is observed, especially at the initial stage, in the lower part of the reactor the temperature distribution is leveled. Based on this, it can be concluded that the thermal conductivity of the gas phase does not have a strong effect on the temperature distribution over the height of the reactor.

\section{Conclusion}

By varying various technological, design parameters and physical properties, both of the catalyst, which is the main heat transformer in microwave technology, and the physical properties of the reaction mixture, it is possible to achieve optimal conditions for carrying out chemical transformations in a microwave field, both from the standpoint of the thermodynamics of a electrodynamic reactor and positions of the chemistry of specific transformations.

The presented model makes it possible to determine the optimal values of the parameters of electrodynamic reactors at a given value of the yield of the target products, to evaluate the influence of various technological parameters of reaction devices on the thermal efficiency of the processes taking place in them, taking into account the supply of heat to the reaction zone by means of microwave.

The electrodynamic reactor is fundamentally different from the reactors currently operating in industry in the way of supplying energy to the reaction zone, which significantly reduces energy consumption, simplifies the process control and increases the efficiency of the installation.

\section{References:}

[1] Rakhmankulov D.L., Bikbulatov I.Kh., Shulaev N.S., Shavshukova S.Yu., Microwave Radiation and Intensification of Chemical Processes, M.: Chemistry, 2003.

[2] Shulaeva E.A., Improvement of technological production on the basis of modeling the processes of chemical technologies: monograph, Ufa: Publishing House "Oil and Gas Business", 2018.

[3] Shulaeva E.A., Shulaev N.S., Calculation and Modeling of the Temperature Conditions of Electrodynamic Chemical Reactors, Chemical and Petroleum Engineering, Vol.52, No.1, 2016, pp. 3-9.

[4] Janke E., Emde F., Lesh F., Special functions: formulas, graphs, tables, M.: Science, 1977.
[5] Bikbulatov I.Kh., Daminev R.R., Shulaev N.S., Shulaev S.N., 1998, Pat. of the Russian Federation No. 2116826, appl. 27.01.1997, publ. 10.08.1998.

[6] Bikbulatov I.Kh., Daminev R.R., Shulaev N.S., Shulaeva E.A., Feoktistov L.R., Modeling the Process of Dehydrogenation of Butenes in an Electrodynamic Catalytic Reactor, Butlerov Communications, Vol.24, No.1, 2011, pp. 99104.

[7] N. Golden Stepha, D. Kavin Jacob, Numerical simulation for convective heat and mass transfer effect of micropolar nanofluid flow with Variable Viscosity and radiation, WSEAS Transactions on Heat and Mass Transfer, Volume 16, 2021, pp. 29-33.

[8] Yedilkhan Amirgaliyev, Murat Kunelbayev, Aliya Kalizhanova, Beibut Amirgaliyev, Ainur Kozbakova, Omirlan Auelbekov, Nazbek Kataev, The study of thermal and convective heat transfer in flat solar collectors, WSEAS Transactions on Heat and Mass Transfer, Volume 15, 2020, pp. 55-63.

\section{Contribution of Individual Authors to the Creation of a Scientific Article (Ghostwriting Policy)}

Ekaterina A. Shulaeva developed a mathematical model and performed numerical modeling of an electrodynamic reactor.

\section{Creative Commons Attribution License 4.0 (Attribution 4.0 International, CC BY 4.0)}

This article is published under the terms of the Creative Commons Attribution License 4.0 https://creativecommons.org/licenses/by/4.0/deed.en US 